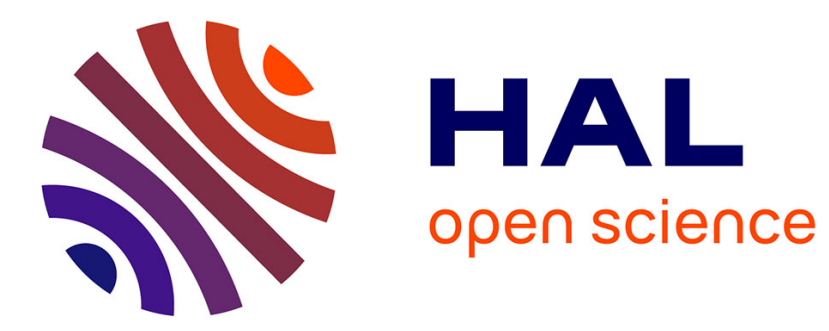

\title{
Optimal cavity shape design for acoustic liners using Helmholtz equation with visco-thermal losses
}

Gilles Tissot, Robin Billard, Gwenael Gabard

\section{To cite this version:}

Gilles Tissot, Robin Billard, Gwenael Gabard. Optimal cavity shape design for acoustic liners using Helmholtz equation with visco-thermal losses. Journal of Computational Physics, 2020, 402, pp.109048. 10.1016/j.jcp.2019.109048 . hal-02335784

\section{HAL Id: hal-02335784 \\ https://hal.inria.fr/hal-02335784}

Submitted on 28 Oct 2019

HAL is a multi-disciplinary open access archive for the deposit and dissemination of scientific research documents, whether they are published or not. The documents may come from teaching and research institutions in France or abroad, or from public or private research centers.
L'archive ouverte pluridisciplinaire HAL, est destinée au dépôt et à la diffusion de documents scientifiques de niveau recherche, publiés ou non, émanant des établissements d'enseignement et de recherche français ou étrangers, des laboratoires publics ou privés. 


\title{
Optimal cavity shape design for acoustic liners using Helmholtz equation with visco-thermal losses
}

\author{
Gilles Tissot $^{\mathrm{a}, \mathrm{b}}$, Robin BillarD ${ }^{\mathrm{a}}$, Gwénaël GABARD ${ }^{\mathrm{a}}$ \\ ${ }^{a}$ Laboratoire d'Acoustique de l'Université du Mans, av. Olivier Messiaen, 72085 Le \\ Mans, France \\ ${ }^{b}$ INRIA Rennes Bretagne Atlantique, IRMAR - UMR CNRS 6625, av. Général Leclerc, \\ 35042 Rennes, France
}

\begin{abstract}
This paper presents a shape optimisation strategy to design a cavity for acoustic liners, that approaches at best a target impedance over a given frequency range, penalising too large shape displacements from an initial guess. A model based on the Helmholtz equation is used, where the viscothermal losses are taken into account by an equivalent impedance boundary condition. Using an adjoint-based method, the gradient of the cost functional with respect to shape variations is calculated, and regularised by a Sobolev gradient. A finite element method is employed with XFEM cut elements, that allows to consider an immersed boundary which is localised by a level-set function. We show that with this method, we are able to obtain a cavity shape leading to an almost perfect absorption for a frequency in the prescribed optimisation range.
\end{abstract}

Keywords: Shape optimisation, Acoustic liners, Impedance matching, Regularisation 


\section{Introduction}

Acoustic liners are integrated in nacelles of aircraft engines in order to absorb noise coming from the fan or the combustion chamber. Constraints on treatment size, weight, and frequency range become extremely restrictive, especially when the trend is the design of higher dilution rate engines. Indeed, in that case, the noise is shifted toward low frequencies, requiring thicker liners, while in contrary thinner treatments are desired. The standard singledegree-of-freedom liners are composed of a honeycomb structure covered by a perforated plate. The principle is to generate a resonance in the cavity, that induces large velocities through the perforations, where viscous dissipations take place, resulting in sound absorption. The context of the present study is to search for optimal cavity shapes in order to absorb as much as possible at low frequencies. We adopt then a strategy of shape optimisation.

Shape optimisation has become popular in various domains, such as in elasticity (Díaaz and Kikuchi, 1992; Ma et al., 1994; Allaire et al., 2004, 2005; Achtziger and Kočvara, 2007), aerodynamics (Jameson, 2003; Sonntag et al., 2016) or optics (Jensen and Sigmund, 2005; Wang et al., 2011). In acoustics some studies were performed, mainly to find optimal horns (Bängtsson et al., 2003; Udawalpola and Berggren, 2008; Wadbro et al., 2010; Udawalpola et al., 2011; Bernland et al., 2018), to optimize Helmholtz resonators (Caeiro et al., 2017) or for room acoustics (Dühring et al., 2008; Desai et al., 2018). In acoustics, the use of the Helmholtz equation is convenient for its simplicity and its relatively low numerical cost. However, it neglects viscous losses and doesn't allow for the exploration of the absorption mechanisms. On the

other hand, the use of the full linearised Navier-Stokes equations, as in Caeiro 
et al. (2017), can become extremely expensive in terms of numerical costs. We propose in the present study to develop a shape optimisation strategy with the model introduced by Berggren et al. (2018), that is a Helmholtzlike equation with a model of losses. This model is similar to the study of Bossart et al. (2003) and assumes that most of the visco-thermal losses take place within the acoustic boundary layer. Based on an integral across the acoustic boundary layer, and assuming that it is infinitely thin with small wall curvatures, it leads to an equivalent impedance boundary condition mimicking the visco-thermal losses.

A recent study of Andersen et al. (2019) handles a similar objective than the present paper optimising Helmholtz resonators for maximising the absorption coefficients but for a transmission problem and not in normal incidence. Their approach is complementary compared to our study since they use a BEM formulation and the shape is parametrised by cubic splines and a single frequency is targeted, while we don't assume any parametrisation due to the XFEM formulation.

A common difficulty in shape optimisation is that it can yield shapes that are very efficient, but so complex that they are impossible to manufacture. Inspired by the work of Allaire et al. (2016) and the definition of the shape distance function, we propose a Tikhonov regularisation (Tikhonov and Arsenin, 1977) by penalising shape displacements from a given initial guess.

In section 2 we present the model and we define the variables necessary for the optimisation. In section 3 we set up the optimisation problem in order to match a target impedance in a given frequency range. We detail how the shape derivative is determined by adjoint method. Moreover, we present the 
Sobolev gradient regularisation necessary to seek the optimal shape in a class of regular solutions. In section 4 the finite element discretisation is presented with the XFEM cut elements strategy. We detail the transport and the regularisation of the level set function. Finally in section 5, the application of the methodology on the liner problem is presented.

\section{Problem formulation}

We consider the domain $\Omega$ representing a cylindrical cavity with radius $R$ and depth $D$, a single perforation with diameter $d$ and thickness $e$ and the exterior domain of length $L$. The geometry is axisymmetric with cylindrical coordinates $\boldsymbol{x}=(r, z)^{T}$ and $\mathrm{d} \boldsymbol{x}=(r \mathrm{~d} r, \mathrm{~d} z)^{T}$. All quantities are nondimensionalised by the reference length $L_{\text {ref }}$, the sound speed $c_{\infty}$ and the fluid density $\rho_{\infty}$. Pressure $p$ is nondimensionalised by $\rho_{\infty} c_{\infty}^{2}$. Figure 1 represents the geometry, with $\Gamma_{w}$ the wall boundary, $\Gamma_{s}$ the free slip boundary and the symmetry axis and $\Gamma_{Z}$ the impedance boundary condition where an incom-

ing plane wave is imposed. In this study, we consider the response of the treatment to a normal incidence, that is sufficient for a full characterisation under the assumption of a locally reacting liner. With the above hypothesis the behaviour of a single cell is similar to a periodic layout when the percent open area (POA) matches. We define $\Gamma=\Gamma_{w} \cup \Gamma_{s} \cup \Gamma_{Z}$. 


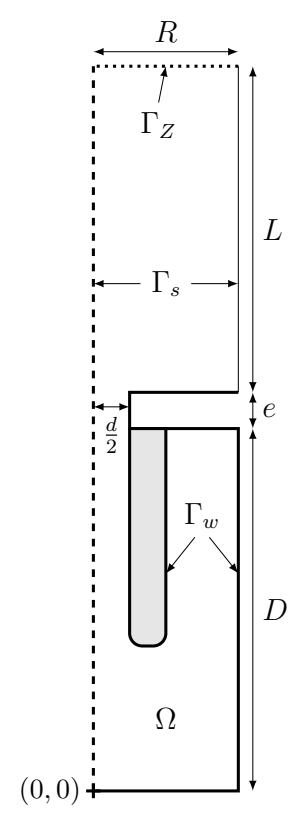

Figure 1: Schematic representation of the domain. The grey zone is the solid part whose shape will be optimised.

We consider the Helmholtz equation in $\Omega$ :

$$
\begin{cases}\Delta p+k^{2} p=0 & \text { in } \Omega \\ \frac{\partial p}{\partial n}=\delta_{v} \frac{i-1}{2} \Delta_{T} p-\delta_{T} k^{2} \frac{(i-1)(\gamma-1)}{2} p & \text { on } \Gamma_{w} \\ \frac{\partial p}{\partial n}+i k p=2 i k e^{i k L} & \text { on } \Gamma_{Z} \\ \frac{\partial p}{\partial n}=0 & \text { on } \Gamma_{s}\end{cases}
$$

where $\boldsymbol{n}(\boldsymbol{x})$ is the local, outward, unit normal. We consider at the walls $\left(\Gamma_{w}\right)$ the condition presented in Berggren et al. (2018). This condition assumes that visco-thermal losses are localised in the acoustic boundary layer of an isothermal wall. Clearly, we neglect the losses in the bulk of the fluid, and losses that could be induced by the shear of the velocity perturbation in 
case of flow separation. Such a separation may appear for instance, even in the linear regime, at sharp corners at the perforation exit. These losses have been quantified in a case of macro- and micro-perforated liners in Billard et al. (2019). For the goal of the present study, it can be considered small compared to the overall losses. The boundary condition is determined by an integral in the wall-normal direction of the acoustic boundary layer equations (Berggren et al., 2018). It assumes an infinitely thin boundary layer, and a small wall curvature. We assume then that the optimal shape will be smooth enough (small curvature) with walls that are not too close from each other, so that the boundary layers do not interact. We rely on regularisation techniques explained in section 3 in order to avoid as much as possible such situations out of modelling hypothesis.

In $(1)_{2}, \Delta_{T}$ is the tangential Laplacian defined such that

$$
\Delta p=\Delta_{T} p+\frac{\partial^{2} p}{\partial n^{2}}+\left(\nabla_{T} \cdot \boldsymbol{n}\right) \frac{\partial p}{\partial n} .
$$

The tangential gradient operator $\nabla_{T}$ is defined such that

$$
\nabla p=\nabla_{T} p+\frac{\partial p}{\partial n} \boldsymbol{n}
$$

Moreover, we have the viscous and thermal acoustic boundary layer thicknesses

$$
\delta_{v}=\sqrt{\frac{2}{\omega R e_{a}}} \quad ; \quad \delta_{T}=\sqrt{\frac{2}{\omega R e_{a} P r}},
$$

with $R e_{a}=c_{\infty} L_{\text {ref }} / \nu_{\infty}$ the acoustic Reynolds number and $\operatorname{Pr}=\frac{\nu_{\infty} c_{p \infty}}{\rho_{\infty} \kappa_{\infty}}$ the Prandtl number. $\nu_{\infty}, c_{p \infty}$ and $\kappa_{\infty}$ are respectively the kinematic viscosity, 
the thermal capacity and thermal conductivity. An impedance boundary condition is considered for $\Gamma_{Z}$ at the entrance of the tube $\left(z=L_{Z}=D+e+L\right)$ enforcing an incoming unitary normal plane wave. We assume the angular frequency $\omega=k c$ to be below the cut-off frequency in the exterior domain, with $c$ the sound speed and $k$ the associated wavenumber. The length $L$ of the exterior domain is chosen sufficiently large so that evanescent waves generated at the hole will have completely decayed at the upper boundary of the computational domain.

From the pressure on $\Gamma_{Z}$ one can recover the reflection coefficient at $z=L_{t}=D+e:$

$$
R_{f}=\left(\bar{p}-e^{i k L}\right) e^{i k L}
$$

with $\bar{p}=\frac{2}{R^{2}} \int_{\Gamma_{Z}} p(r, L) r \mathrm{~d} r$ the average pressure on the boundary $z=L_{Z}$. The impedance of the treatment at $z=L_{t}$ is then

$$
Z=\frac{1+R_{f}}{1-R_{f}}=\frac{e^{-i k L}-e^{i k L}+\bar{p}}{e^{-i k L}+e^{i k L}-\bar{p}} .
$$

\section{Optimisation problem}

\subsection{Impedance matching}

The objective is to modify the shape of the cavity, i.e. the domain $\Omega_{c}=$ $\{\boldsymbol{x}=(r, z) \in \Omega \mid z<D\}$, in order to match a given target impedance $Z_{T}(k)$ for a given frequency range $k \in\left[k_{1}, k_{2}\right]$. In normal incidence, perfect absorption is obtained for $Z_{T}=1$, but other values can be chosen for the target impedance depending on the conditions of integration of the liner. We consider the boundary $\Gamma_{\theta} \subset \Gamma_{w}$ of $\Omega_{c}$, that we allow to move during the optimisation, and that will be further modelled by an immersed boundary. 
It is materialised by the grey region in figure 1 . We define the cost functional as follows

$$
\mathcal{J}(\Omega, p)=\frac{1}{2} \int_{k_{1}}^{k_{2}}\left|Z(k)-Z_{T}(k)\right|^{2} \mathrm{~d} k .
$$

The problem can be formulated as seeking $\Omega$ that minimises $(7)$ under the constraint (1).

To transform this constrained optimisation problem into an unconstrained optimisation problem, we define the Lagrangian

$$
\mathcal{L}(\Omega, p, \lambda)=\mathcal{J}(p)-\operatorname{real}\left(\int_{k_{1}}^{k_{2}}\left(\lambda, \Delta p+k^{2} p\right) \mathrm{d} k\right)
$$

with $(a, b)=\int_{\Omega} a^{*} b \mathrm{~d} \boldsymbol{x}$ and $a^{*}$ denotes the conjugate of $a . \lambda$ is the Lagrange multiplier defined to enforce the constraint $(1)_{1}$. Other constraints $(1)_{2}$ to $(1)_{4}$ are enforced by substitution of the boundary terms in the calculations, as it is detailed in Appendix A.1. Then, we differentiate (8) with respect to each variable $(\Omega, p, \lambda)$, the corresponding derivatives are equal to zero at the minimum of $\mathcal{J}$.

The differentiation with respect to $\lambda$ leads to equation $(1)_{1}$, that proves that the constraint is respected when the minimum is reached. The Fréchet 
derivative with respect to $p$ leads to the following adjoint equation

$$
\begin{cases}\Delta \lambda+\left(k^{*}\right)^{2} \lambda=0 & \boldsymbol{x} \in \Omega \\ \frac{\partial \lambda}{\partial n}+\delta_{v} \frac{i+1}{2} \Delta_{T} \lambda-\delta_{T}\left(k^{*}\right)^{2} \frac{(i+1)(\gamma-1)}{2} \lambda=0 & \boldsymbol{x} \in \Gamma_{w} \\ \frac{\partial \lambda}{\partial n}-i k^{*} \lambda=\frac{4}{R^{2}} \frac{e^{i k L} j(p, k)}{\left(\left(e^{-i k L}+e^{i k L}-\bar{p}\right)^{2}\right)^{*}} & \boldsymbol{x} \in \Gamma_{Z} \\ \frac{\partial \lambda}{\partial n}=0 & \boldsymbol{x} \in \Gamma_{s} .\end{cases}
$$

The shape sensitivity of $\mathcal{J}$ is determined by vanishing the shape sensitivity of $\mathcal{L}$, following the formalism presented in Allaire et al. (2004). Let $\delta \boldsymbol{\theta}(\boldsymbol{x})$ be a smooth $^{1}$ displacement direction field of $\Omega$ and $(f, g)$ two scalar objective functions. We define the cost functional

$$
\mathcal{J}(\Omega)=\int_{\Omega} f(\boldsymbol{x}) \mathrm{d} \boldsymbol{x}+\int_{\Gamma} g(\boldsymbol{x}) \mathrm{d} \boldsymbol{x} .
$$

The shape derivative $\nabla_{\delta \boldsymbol{\theta}} \mathcal{J}$ of the movable boundary $\Gamma_{\theta}$, defined in a weak sense by

$$
\left(\nabla_{\delta \boldsymbol{\theta}} \mathcal{J}, \delta \boldsymbol{\theta} . \boldsymbol{n}\right)_{\Gamma_{\boldsymbol{\theta}}}=\lim _{\epsilon \rightarrow 0} \frac{\mathcal{J}(\Omega+\epsilon \delta \boldsymbol{\theta})-\mathcal{J}(\Omega)}{\epsilon} \quad \forall \delta \boldsymbol{\theta}
$$

is equal to

$$
\left(\nabla_{\delta \boldsymbol{\theta}} \mathcal{J}, \delta \boldsymbol{\theta} \cdot \boldsymbol{n}\right)_{\Gamma_{\theta}}=\int_{\Gamma_{\theta}} \delta \boldsymbol{\theta}(\boldsymbol{x}) \cdot \boldsymbol{n}(\boldsymbol{x})(f(\boldsymbol{x})+\nabla g \cdot \boldsymbol{n}+H g) \mathrm{d} \boldsymbol{x} \quad \forall \delta \boldsymbol{\theta}
$$

where $H$ is the local curvature of the boundary $H=\operatorname{div}(\boldsymbol{n})$. Since shape

\footnotetext{
${ }^{1}$ According to Allaire et al. (2004), $\delta \boldsymbol{\theta}(\boldsymbol{x}) \in W^{1, \infty}\left(\mathbb{R}^{2} ; \mathbb{R}^{2}\right)$ and $(f, g) \in W^{1,1}\left(\mathbb{R}^{2}\right) \times$ $W^{2,1}\left(\mathbb{R}^{2}\right)$.
} 
derivatives involve only quantities at the boundary, the real inner product used to identify the gradient is $(a, b)_{\Gamma_{\theta}}=\int_{\Gamma_{\theta}} a b \mathrm{~d} \boldsymbol{x}$. Moreover only normal shape displacements matter, this is why it is directly introduced in the weak form. In our case, we obtain

$$
\begin{aligned}
& \left(\nabla_{\delta \boldsymbol{\theta}} \mathcal{L}, \delta \boldsymbol{\theta} \cdot \boldsymbol{n}\right)_{\Gamma_{\theta}}=\left(\nabla_{\delta \boldsymbol{\theta}} \mathcal{J}, \delta \boldsymbol{\theta} \cdot \boldsymbol{n}\right)_{\Gamma_{\theta}} \\
& -\int_{k_{1}}^{k_{2}} \operatorname{Real}\left[\int_{\Gamma_{\theta}}(\delta \boldsymbol{\theta}(\boldsymbol{x}) \cdot \boldsymbol{n}(\boldsymbol{x}))\left(-\nabla \lambda^{*} . \nabla p+k^{2} \lambda^{*} p\right) \mathrm{d} \boldsymbol{x}\right] \mathrm{d} k \\
& -\int_{k_{1}}^{k_{2}} \operatorname{Real}\left[\int_{\Gamma_{\theta}}(\delta \boldsymbol{\theta}(\boldsymbol{x}) \cdot \boldsymbol{n}(\boldsymbol{x}))\left(\frac{\partial}{\partial n}\left(\lambda^{*} \frac{\partial p}{\partial n}\right)+H \lambda^{*} \frac{\partial p}{\partial n}\right) \mathrm{d} \boldsymbol{x}\right] \mathrm{d} k \\
= & 0 .
\end{aligned}
$$

We assume here that $\Gamma_{\theta} \subset \Gamma_{w}$, then $\Gamma_{\theta} \cap \Gamma_{Z}=0$, that means that we are only modifying the shape of the wall. We can note that due to the viscous model, the term $\frac{\partial p}{\partial n}$ does not vanish and the boundary terms should be explicitly computed. We obtain the shape derivative $\nabla_{\delta \boldsymbol{\theta}} \mathcal{J}$

$$
\begin{aligned}
& \nabla_{\delta \boldsymbol{\theta}} \mathcal{J}=\int_{k_{1}}^{k_{2}} \operatorname{Real}\left[\left(-\nabla \lambda^{*} \cdot \nabla p+k^{2} \lambda^{*} p\right)+\right. \\
& \left.\left(\frac{\partial \cdot}{\partial n}+H \cdot\right)\left(-\delta_{v} \frac{i-1}{2} \nabla_{T} \lambda^{*} \cdot \nabla_{T} p-\delta_{T} k^{2} \frac{(i-1)(\gamma-1)}{2} \lambda^{*} p\right)\right] \mathrm{d} k .
\end{aligned}
$$

\subsection{Regularisation}

Shape optimisation suffers intrinsically from the fact that the solution can be arbitrarily complex and then hardly workable. This difficulty can be solved by prescribing an a priori shape contour, from which the solution has to be close in a given sense. Thus, we would like to penalise a too large shape variation from the initial domain $\Omega_{0}$ by Tikhonov regularisation. This 
specification has two main advantages: i) it renders the optimisation problem more convex and avoids unwanted local minima ii) it provides the designer with a tool, embedded in the formulation, to guide the optimisation with a prior information of the desired class of solution. For that, we define the signed distance function to the initial movable boundary $\Gamma_{\theta, 0}$, in a similar manner than in Allaire et al. (2016),

$$
d_{\Omega_{0}}(\boldsymbol{x})= \begin{cases}-d\left(\boldsymbol{x}, \Gamma_{\theta, 0}\right) & \text { if } \boldsymbol{x} \in \Omega_{0} \\ 0 & \text { if } \boldsymbol{x} \in \Gamma \\ d\left(\boldsymbol{x}, \Gamma_{\theta, 0}\right) & \text { if } \boldsymbol{x} \notin \Omega_{0},\end{cases}
$$

with $d(\boldsymbol{x}, \Gamma)$ the Euclidean distance between $\boldsymbol{x}$ and a boundary $\Gamma$ :

$$
d(\boldsymbol{x}, \Gamma)=\min _{\boldsymbol{y} \in \Gamma}|\boldsymbol{x}-\boldsymbol{y}| .
$$

We define the new cost functional regularised by a penalty term as follows

$$
\mathcal{J}_{r}(\Omega, p)=\mathcal{J}(\Omega, p)+\beta \frac{1}{2} \int_{\Gamma_{\theta}} d_{\Omega_{0}}^{2}(\boldsymbol{x}) \mathrm{d} \boldsymbol{x} .
$$

Remarking that $\nabla d_{\Omega_{0}}(\boldsymbol{x})=\boldsymbol{n}(\boldsymbol{x})$ on $\Gamma_{\theta}$, we can deduce that

$$
\begin{aligned}
\left(\nabla_{\delta \boldsymbol{\theta}} \mathcal{J}_{r}, \delta \boldsymbol{\theta} \cdot \boldsymbol{n}\right)_{\Gamma_{\theta}}= & \left(\nabla_{\delta \boldsymbol{\theta}} \mathcal{J}, \delta \boldsymbol{\theta} \cdot \boldsymbol{n}\right)_{\Gamma_{\theta}} \\
& +\beta \int_{\Gamma_{\theta}}(\delta \boldsymbol{\theta}(\boldsymbol{x}) \cdot \boldsymbol{n}(\boldsymbol{x}))\left(d_{\Omega_{0}}(\boldsymbol{x})+H \frac{1}{2} d_{\Omega_{0}}^{2}(\boldsymbol{x})\right) \mathrm{d} \boldsymbol{x} .
\end{aligned}
$$


Thus, we identify

$$
\nabla_{\delta \boldsymbol{\theta}} \mathcal{J}_{r}=\nabla_{\delta \boldsymbol{\theta}} \mathcal{J}+\beta\left(d_{\Omega_{0}}(\boldsymbol{x})+H \frac{1}{2} d_{\Omega_{0}}^{2}(\boldsymbol{x})\right)
$$

Numerically, the level-set functions defined in section 4 are used to approximate $d_{\Omega_{0}}(\boldsymbol{x})$.

The parameter $\beta$ controls how far the optimal shape can be from the initial guess and is determined a posteriori.

\subsection{Descent direction}

For the minimisation, we propose to use a Sobolev gradient descent direction, as in Protas et al. (2004), that has the property to be smoother than the gradient defined in (14). The Sobolev gradient $\nabla_{\delta \boldsymbol{\theta}}^{H^{1}} \mathcal{J}_{r}$ is defined such that for all real function $q$, we have

$$
\left(\nabla_{\delta \boldsymbol{\theta}} \mathcal{J}_{r}, q\right)=\left(\nabla_{\delta \boldsymbol{\theta}}^{H^{1}} \mathcal{J}_{r}, q\right)_{H^{1}}
$$

with $(a, b)_{H^{1}}=\int_{\Omega} a b+l_{\text {Sob }}^{2} \nabla a . \nabla b \mathrm{~d} \boldsymbol{x}$. The parameter $l_{\text {Sob }}$ is a characteristic length associated with the spatial smoothing. We choose $l_{\text {Sob }}=0.05$, corresponding to $10 \%$ of $R$, the radius of the domain. We deduce that

$$
\nabla_{\delta \boldsymbol{\theta}}^{H^{1}} \mathcal{J}_{r}=\left(\frac{1}{1+l_{\mathrm{Sob}}^{2}}\left(\mathbb{I}-l_{\mathrm{Sob}}^{2} \Delta\right)\right)^{-1} \nabla_{\delta \boldsymbol{\theta}} \mathcal{J}_{r}
$$

where $\mathbb{I}$ is the identity operator. The steepest descent direction with Sobolev regularisation is the normal displacement $\delta \boldsymbol{\theta} . \boldsymbol{n}=-\nabla_{\delta \boldsymbol{\theta}}^{H^{1}} \mathcal{J}_{r}$. It is ensured to 
be a descent direction since we have

$$
\begin{aligned}
\mathcal{J}_{r}(\Omega+\epsilon \delta \boldsymbol{\theta}) & =\mathcal{J}_{r}(\Omega)+\epsilon\left(\nabla_{\delta \boldsymbol{\theta}} \mathcal{J}_{r}, \delta \boldsymbol{\theta} . \boldsymbol{n}\right)+\mathcal{O}\left(\epsilon^{2}\right) \\
& =\mathcal{J}_{r}(\Omega)+\epsilon\left(\nabla_{\delta \boldsymbol{\theta}}^{H^{1}} \mathcal{J}_{r}, \delta \boldsymbol{\theta} \cdot \boldsymbol{n}\right)_{H_{1}}+\mathcal{O}\left(\epsilon^{2}\right) \\
& =\mathcal{J}_{r}(\Omega)-\epsilon\left\|\nabla_{\delta \boldsymbol{\theta}}^{H^{1}} \mathcal{J}_{r}\right\|_{H_{1}}^{2}+\mathcal{O}\left(\epsilon^{2}\right) .
\end{aligned}
$$

We can then always find an $\epsilon$ small enough such that $\mathcal{J}_{r}(\Omega+\epsilon \delta \boldsymbol{\theta})<\mathcal{J}_{r}(\Omega)$. Numerically, a suitable value of $\epsilon$ is found using a backtracking Armijo algorithm (Armijo, 1966). The optimisation procedure is described in the flow chart in figure 2 .

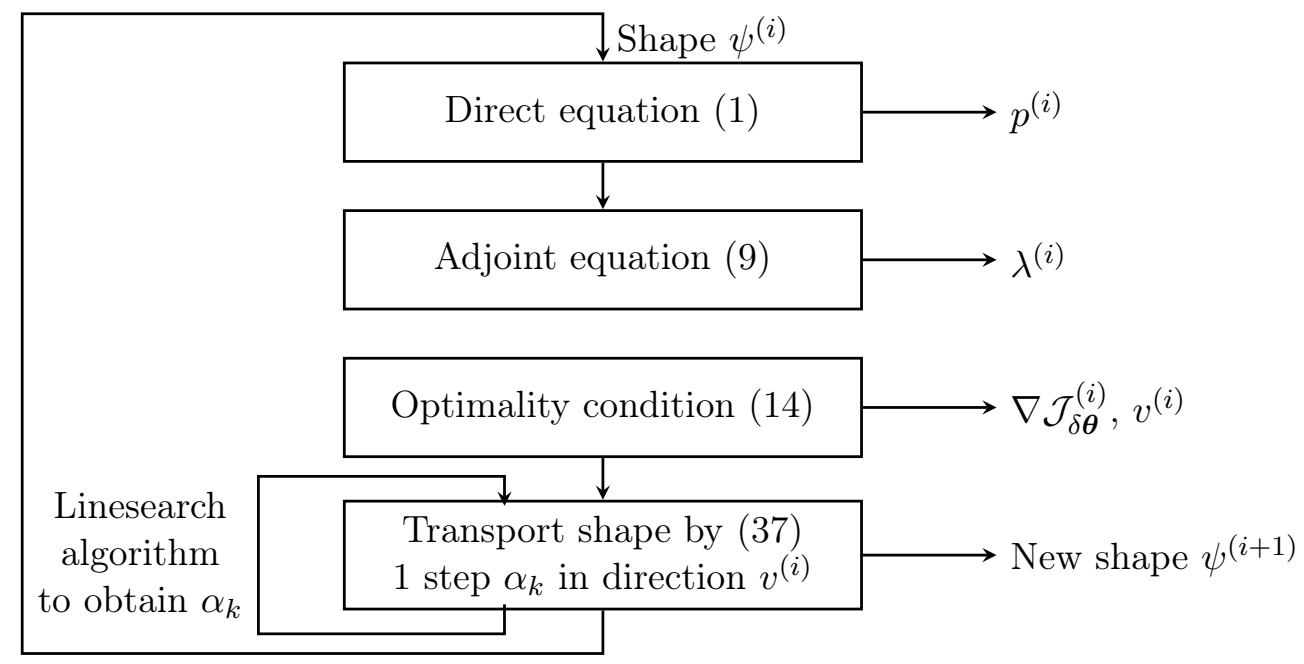

Figure 2: Flow chart of the shape optimisation procedure. The transport of the shape is detailed in section 4 . 


\section{Numerical methods}

\subsection{Discrete model}

We consider a finite element approximation. To avoid a remeshing procedure during the minimisation, an immersed boundary is taken into account by XFEM cut elements (Moës et al., 1999; Legrain et al., 2012; Burman et al., 2015). Defining the test function $q$, we consider the following variational formulation for the direct problem (1):

$$
\begin{aligned}
& \int_{\Omega}-\nabla p \cdot \nabla q+k^{2} p q \mathrm{~d} \boldsymbol{x}+\int_{\Gamma_{w}}-\delta_{v} \frac{i-1}{2} \nabla_{T} p . \nabla_{T} q-\delta_{T} k^{2} \frac{(i-1)(\gamma-1)}{2} p q \mathrm{~d} l \\
& +\int_{\Gamma_{Z}}-i k p q+2 i k e^{i k L} q \mathrm{~d} l=0 .
\end{aligned}
$$

Boundary conditions in (1) are enforced by substituting $\frac{\partial p}{\partial n}$ in the contour terms. For the adjoint problem (9), we define the test function $\mu$ and we obtain by the same procedure

$$
\begin{aligned}
& \int_{\Omega}-\nabla \lambda \cdot \nabla \mu+\left(k^{*}\right)^{2} \lambda \mu \mathrm{d} \boldsymbol{x} \\
& +\int_{\Gamma_{w}} \delta_{v} \frac{i+1}{2} \nabla_{T} \lambda . \nabla_{T} \mu+\delta_{T}\left(k^{*}\right)^{2} \frac{(i+1)(\gamma-1)}{2} \lambda \mu \mathrm{d} l \\
& +\int_{\Gamma_{Z}} i k^{*} \lambda \mu+\frac{4}{R^{2}} \frac{e^{i k L} j(p, k)}{\left(\left(e^{-i k L}+e^{i k L}-\bar{p}\right)^{2}\right)^{*}} \mu \mathrm{d} l=0 .
\end{aligned}
$$

More compactly we write for the direct problem

$$
\boldsymbol{K} \boldsymbol{p}+i k \boldsymbol{D} \boldsymbol{p}-k^{2} \boldsymbol{M} \boldsymbol{p}=i k e^{i k L} \boldsymbol{f}
$$


with $\boldsymbol{p}$ the vector of the degrees of freedom associated with $p$ and

$$
\begin{aligned}
(\boldsymbol{K})_{i j} & =\int_{\Omega} \nabla \phi_{i} . \nabla \phi_{j} \mathrm{~d} \boldsymbol{x}+\int_{\Gamma_{w}} \delta_{v} \frac{i-1}{2} \nabla_{T} \phi_{i} . \nabla_{T} \phi_{j} \mathrm{~d} l, \\
(\boldsymbol{D})_{i j} & =\int_{\Gamma_{z}} \phi_{i} \phi_{j} \mathrm{~d} l, \\
(\boldsymbol{M})_{i j} & =\int_{\Omega} \phi_{i} \phi_{j} \mathrm{~d} \boldsymbol{x}-\int_{\Gamma_{w}} \delta_{T} \frac{(i-1)(\gamma-1)}{2} \phi_{i} \phi_{j} \mathrm{~d} l, \\
(\boldsymbol{f})_{i} & =\int_{\delta \Omega_{L}} 2 \phi_{i} \mathrm{~d} l .
\end{aligned}
$$

Cut elements can induce numerical oscillations due to ill-conditioning of the mass matrix when the boundary passes very close to a mesh node. Indeed, in that situation, the size of some elements can become very small. Then, a stabilisation term $\boldsymbol{S}$ is added consistently with the one proposed by Burman (2010),

$$
(\boldsymbol{K}+\boldsymbol{S}) \boldsymbol{p}+i k \boldsymbol{D} \boldsymbol{p}-k^{2} \boldsymbol{M} \boldsymbol{p}=i k e^{i k L} \boldsymbol{f}
$$

such that

$$
(\boldsymbol{S})_{i j}=\sum_{F_{k} \in F_{\text {cut }}} \int_{F_{k}} \sigma_{k} \llbracket \nabla \phi_{i} \rrbracket_{F_{k}} \cdot \llbracket \nabla \phi_{j} \rrbracket_{F_{k}} \mathrm{~d} l,
$$

where $F_{\text {cut }}$ is the ensemble of all edges belonging to a cut element and $\llbracket \cdot \rrbracket_{F_{k}}$ represents the jump of a quantity across an element edge. We choose a constant $\sigma_{k}=10^{-3}$ over the computational domain. In practice, this parameter can be drastically increased without affecting much the solution, but we preferred here to select a value as small as possible to avoid ill-conditioning effects. 
For the adjoint equation, we have

$$
(\boldsymbol{K}+\boldsymbol{S})^{*} \boldsymbol{\lambda}-i k^{*} \boldsymbol{D}^{*} \boldsymbol{\lambda}-\left(k^{*}\right)^{2} \boldsymbol{M}^{*} \boldsymbol{\lambda}=\frac{2}{R^{2}} \frac{e^{i k L} j(p, k)}{\left(\left(e^{-i k L}+e^{i k L}-\bar{p}\right)^{2}\right)^{*}} \boldsymbol{f}
$$

with $\boldsymbol{\lambda}$ the vector of the degrees of freedom associated with $\lambda$.

\subsection{Level-set transport}

Let us consider a working bounded domain $\mathcal{D}$ in which the open set $\Omega \subset \mathcal{D}$ is immersed. In practice, $\mathcal{D}$ is meshed once for all. In order to locate the immersed boundary, we use a level-set function such that

$$
\begin{cases}\psi=0 & \boldsymbol{x} \in \Gamma_{\theta} \cap \mathcal{D} \\ \psi>0 & \boldsymbol{x} \in \Omega \\ \psi<0 & \boldsymbol{x} \in\left(\mathcal{D} \backslash\left(\Omega \cup \Gamma_{\theta}\right)\right) .\end{cases}
$$

On $\Gamma_{\theta}$, we have the outward normal direction $\boldsymbol{n}=-\frac{\nabla \psi}{|\nabla \psi|}$, and the curvature $H=\operatorname{div}(\boldsymbol{n})$. To move the shape in the direction $\delta \boldsymbol{\theta}$, the following transport equation is used

$$
\frac{\partial \psi}{\partial t}+\boldsymbol{v} \cdot \nabla \psi=0
$$

Since the shape is invariant with respect to a displacement tangential to the boundary, we consider only normal velocities as shape variations $v=\boldsymbol{v} . \boldsymbol{n}$, that leads to the scalar relation

$$
\frac{\partial \psi}{\partial t}+v|\nabla \psi|=0
$$


We recall that the descent direction is

$$
v=\delta \boldsymbol{\theta} . \boldsymbol{n}=-\nabla_{\delta \boldsymbol{\theta}}^{H^{1}} \mathcal{J}_{r} \cdot \boldsymbol{n} .
$$

The transport equation (32) is solved implicitly with a Streamline Upwind Petrov Galerkin (SUPG) scheme (Donea and Huerta, 2003). Using the test function $(w-\tau v \boldsymbol{n} . \nabla w)$, the following weak formulation is obtained:

$\int_{\Omega} \frac{\partial \psi}{\partial t}(w-\tau v \boldsymbol{n} . \nabla w) \mathrm{d} \boldsymbol{x}-\int_{\Omega} v \boldsymbol{n} . \nabla \psi w \mathrm{~d} \boldsymbol{x}+\tau \int_{\Omega}(v \boldsymbol{n} \cdot \nabla \psi)(v \boldsymbol{n} . \nabla w) \mathrm{d} \boldsymbol{x}=0$.

The implicit Euler scheme is used for the time advancement of (34), however the convection direction $\boldsymbol{n}=-\frac{\nabla \psi}{|\nabla \psi|}$ is explicited. This leads to solve at the $i^{\text {th }}$ time step the following semi-discrete relation

$$
\begin{aligned}
& \int_{\Omega} \psi^{(i+1)}\left(w-\tau v^{(i)} \boldsymbol{n}^{(i)} \cdot \nabla w\right) \mathrm{d} \boldsymbol{x}-\Delta t \int_{\Omega} v^{(i)} \boldsymbol{n}^{(i)} \cdot \nabla \psi^{(i+1)} w \mathrm{~d} \boldsymbol{x} \\
& +\Delta t \tau \int_{\Omega}\left(v^{(i)} \boldsymbol{n}^{(i)} \cdot \nabla \psi^{(i+1)}\right)\left(v^{(i)} \boldsymbol{n}^{(i)} \cdot \nabla w\right) \mathrm{d} \boldsymbol{x} \\
& =\int_{\Omega} \psi^{(i)}\left(w-\tau v^{(i)} \boldsymbol{n}^{(i)} \cdot \nabla w\right) \mathrm{d} \boldsymbol{x} .
\end{aligned}
$$

Following Donea and Huerta (2003), we choose $\tau=\frac{1}{2} \frac{\beta h}{|v|}$, with $\beta=$ $\operatorname{coth}(P e)-\frac{1}{P e}, P e=5$ and $h$ the local element size.

\subsection{Level-set regularisation}

Beside localising the shape boundary, another role of the level set is to give access to the signed distance function $d_{\Omega_{0}}(\boldsymbol{x})$. A way of doing that is to solve the pseudo-temporal equation (see for instance Osher and Fedkiw, 
2001; Allaire et al., 2004):

$$
\frac{\partial \psi}{\partial t}+\operatorname{sign}\left(\psi_{0}\right)(|\nabla \psi|-1)=0
$$

where $\psi_{0}$ is the value of the level set before regularisation. The steady solution of this equation is the distance to the iso-contour $\psi_{0}=0$, i.e. to $\Gamma_{\theta}$. For this reason, we replace in $(17) d_{\Omega_{0}}(\boldsymbol{x})$ by $\psi-\psi_{c i}$, with $\psi_{c i}$ the level set at the initial condition, regularised by (36). These quantities indeed correspond to $d_{\Omega_{0}}(\boldsymbol{x})=-\psi_{0}$ on $\Gamma_{\theta}$, i.e. where they are evaluated.

Moreover, the level-set can become stiff during the transport process. This effect can lead to inaccurate gradients computations. To cope this difficulty, few time steps of this pseudo-temporal equation are performed at each iteration of the optimisation.

Solutions of equation (36) are not regular. To illustrate this, for a onedimensional equation with two wall-points in $\Gamma_{\theta}$, a discontinuity of the gradient appears at the mid-point between the two points. In order to obtain a more regular level-set function, we add a dissipative term that keeps consistency of the steady state to the initial wall location. Inspired by the diffusive level-set regularisation proposed by Li et al. (2010), we propose to solve

$$
\frac{\partial \psi}{\partial t}+\operatorname{sign}\left(\psi_{0}\right)(|\nabla \psi|-1)=\nu \operatorname{div}\left(d_{p}(|\nabla \psi|) \nabla \psi\right)
$$

with

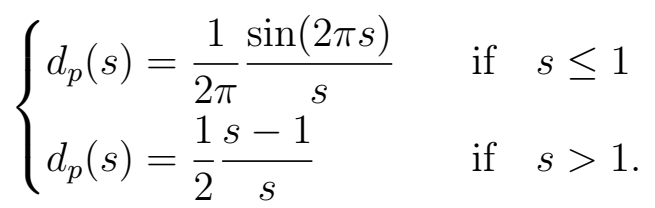


This kernel is designed so that when a steady state is reached $(|\nabla \psi|=1)$, the diffusive term vanishes. This property ensures the consistency of the wall location. It is moreover anti-dissipative when $\frac{1}{2}<|\nabla \psi|<1$, and dissipative otherwise. Increasing $\nu$ improves the regularity of the shape, but, despite the fact that this diffusive term is designed in order to preserve the shape location, increasing $\nu$ too much leads to rounding of zones that have high curvature. In the present case, $\nu=10^{-2}$ is a good trade-off to conserve the initial shape. This effect is visible in the results in section 5.2.

Equation (37) is solved implicitly with a SUPG stabilisation. The weak formulation is detailed in Appendix A.2. An implicit Euler scheme is used and the convection direction $\boldsymbol{n}=-\frac{\nabla \psi}{|\nabla \psi|}$ and the diffusion coefficient $p(|\nabla \psi|)$ are explicited as detailed in Appendix A.2. We perform a single time step per iteration with $\Delta t=10^{-3}$, and $\tau=0.16 h$. This choice of parameters leads to fairly regular level-set functions.

\section{Results}

\subsection{General settings}

The domain considered has a cavity depth $D=2.32$, a thickness $e=0.11$, a hole diameter $d=0.25$, a tube length $L=5$ and a cell radius is $R=0.5$. The domain is discretised by $59352 P 2$ elements, leading to 117427 degrees of freedom. The Reynolds number is $R e_{a}=1.85 \cdot 10^{5}$ and the Prandtl number is $\operatorname{Pr}=0.7$. The orders of magnitude of these parameters are representative of realistic values found for acoustic liners integrated in aircraft engines. For instance in the case of a $19 \mathrm{~mm}$ cavity depth and $c_{\infty}=340 \mathrm{~m} \mathrm{~s}^{-1}$, the initial resonance frequency of approximately $\omega=0.3$ corresponds to a dimensional 
frequency of $2 \mathrm{kHz}$. In that case, the target frequency range around $\omega=0.09$ corresponds to a frequency of $640 \mathrm{~Hz}$. We start with an initial guess for the shape shown in figure 3(a). This shape corresponds to a simple additional tube that could be added to the treatment and that we would like to reshape. This kind of device has the effect of moving the resonance towards lower frequencies. This is done by lengthening the neck of the Helmholtz resonator and by enlarging the cavity in the peripheral region outside the tube. A thin tube would not penalise significantly the cavity volume in an axisymmetric geometry, in favor of a long neck, thus leading to a low resonance frequency. We want to modify the shape in order to decrease even further the resonance frequency, and obtain perfect absorption. Then, we set the target impedance to $Z_{T}=1$, corresponding to a perfect absorption, over the frequency range $k \in[0.08: 0.1]$, slightly lower than the resonance of the initial guess.

\subsection{Impact of the level-set regularisation}

The initial shape is defined by an analytical expression of the level set based on a linear combination of hyperbolic tangent functions. The first step is to regularise the level set by pseudo-time integration of equation (37). Integration of 200 time steps with $\nu=10^{-2}$ leads to the shape in figure 3(b). We can observe the conservation of the shape, with just some slight distortions where there are large curvatures. Figure 4 shows the value of the level-set function $\psi$ at the slice $z=1$ before and after regularisation. We can see that, as expected, the regularised function approximates well the opposite of the signed wall-distance function $d_{\Omega_{0}}(\boldsymbol{x})$. The regularised function figure $3(\mathrm{~b})$ is taken later as initial condition of the optimisation problem and as reference level-set for the penalty term in equation (17). 


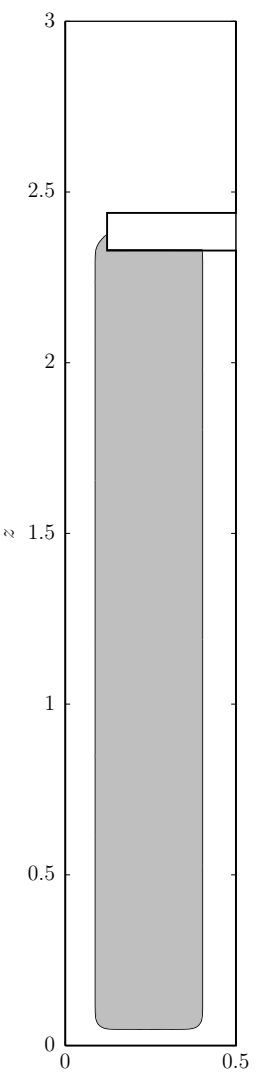

(a) Before regularisation.

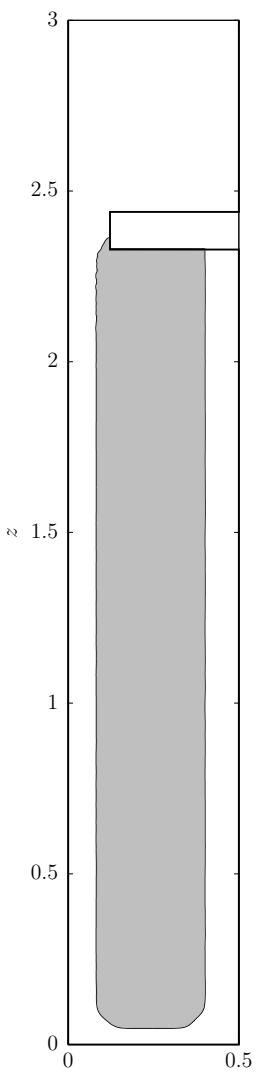

(b) After regularisation, $\nu=10^{-2}$.

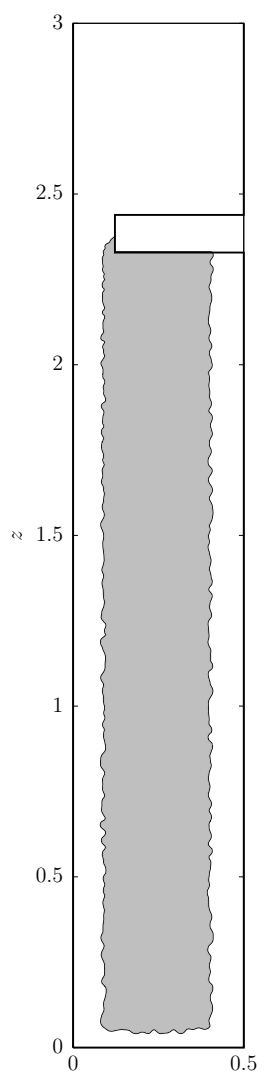

(c) After regularisation, $\nu=0$.

Figure 3: Regularisation of the initial level-set performed with $\nu=10^{-2}$ shown converged after 200 pseudo-time steps and with $\nu=0$ after 25 pseudo-time steps. The shape does not converge with $\nu=0$. 


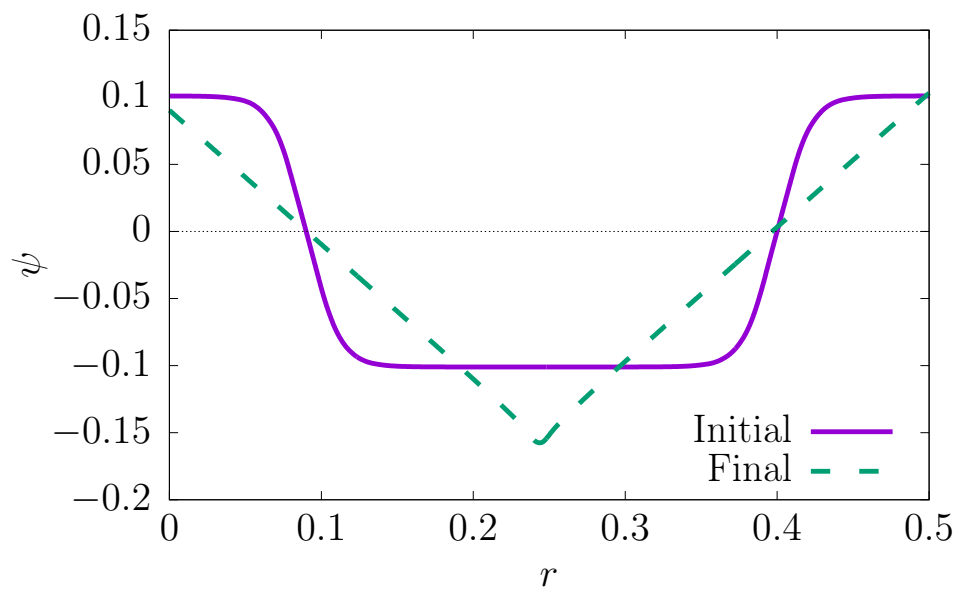

Figure 4: Level-set function $\psi$ before an after regularisation. The slice $z=1$ is displayed.

In order to see the importance of the diffusive term in (37), the same procedure has been performed for $\nu=0$ that leads to the irregular shape in figure 3(c) after only 25 iterations. Further integrating in time amplifies these irregularities.

\subsection{Effect of the ghost penalty}

Before presenting the results of the shape optimisation, we illustrate the importance of the ghost penalty term $S$ in the matrix assembling by comparing the first iteration of the optimisation with $\sigma_{k}=10^{-3}$ and $\sigma_{k}=0$, i.e. with and without ghost penalty respectively. The Sobolev gradient $\nabla \mathcal{J}_{\delta \boldsymbol{\theta}}^{H_{1}}$ at the first iteration is shown in figure 5. We can see in figure 5(a) that there are very large local fluctuations. It can be seen (figure 5(c)) that the singularity is localised at a point where the boundary passes very close to a vertex of the mesh. Moreover, the regularity of the solutions with the ghost penalty term (figures 5(b) and 5(d)) illustrates the benefit of this penalty in the XFEM formulation. 


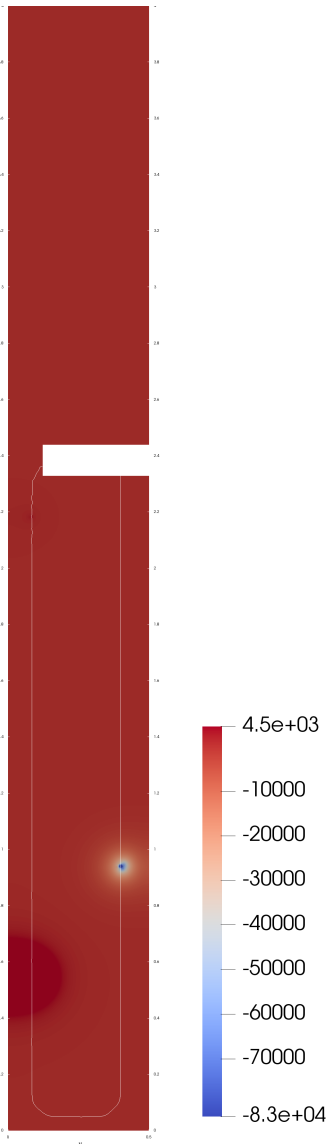

(a) Without ghost penalty.

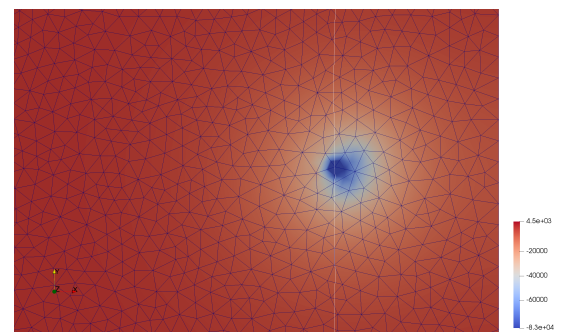

(c) Without ghost penalty, zoom around $(0.4,0.95)$.

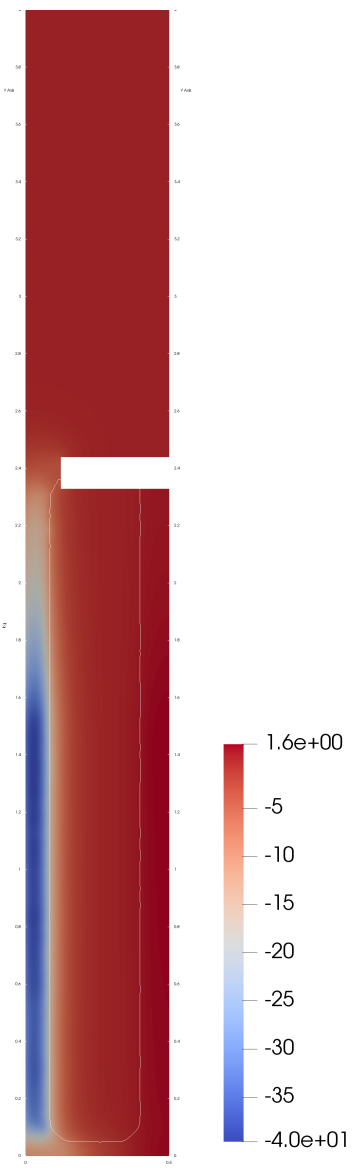

(b) With ghost penalty.

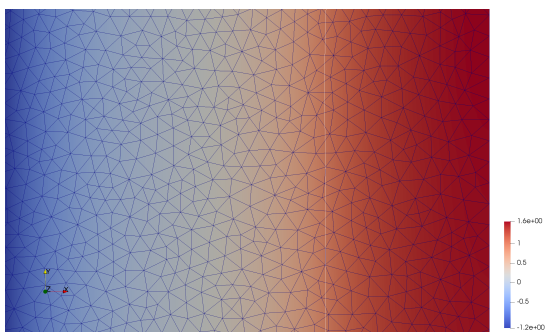

(d) With ghost penalty, zoom around $(0.4,0.95)$.

Figure 5: Effect of the regularisation by ghost penalty by comparing with regularisation $\left(\sigma_{k}=10^{-3}\right)$ and without $\left(\sigma_{k}=0\right)$. Maps are coloured by Sobolev gradient $\nabla \mathcal{J}_{\delta \boldsymbol{\theta}}^{H_{1}}$ at the first iteration of the optimisation problem. The white line represents the shape position. 


\subsection{Shape optimisation}

The parameters used for the optimisation are the following. A tolerance of $10^{-6}$ on variations of $J_{r}$ and of $10^{-3}$ on the $L^{2}$ norm of the gradient define the stopping criteria. The linesearch algorithm starts with an initial step of $\alpha_{0}=2 \cdot 10^{-3}$ with a relaxation parameter $\gamma=0.5$ and an Armijo condition tolerance of $\epsilon_{\text {Armijo }}=0.1$ such that the step is accepted if $\mathcal{J}_{r}^{i}-\mathcal{J}_{r}^{i+1}>$ $\epsilon_{\text {Armijo }} \alpha_{i}\left\|\nabla \mathcal{J}_{r}^{i}\right\|_{H_{1}}$ with $\alpha_{i}$ the optimisation step. In order to highlight the effect of the regularisation by penalising the distance to the initial shape, various values of the associated parameter are chosen $\beta=\left\{10^{-1}, 2 \cdot 10^{-1}, 5\right.$. $\left.10^{-1}, 2 \cdot 10^{0}\right\}$.

Convergence of the optimisation problem is illustrated in figure 6 by the cost functional $\mathcal{J}_{r}$ during the iterations for different values of $\beta$. As it can be expected in a Tikhonov regularisation, increasing the regularisation parameter $\beta$ accelerates the convergence toward a larger value of cost functional $\mathcal{J}_{r}$. The optimal shapes obtained are displayed in figure 7. As expected, the

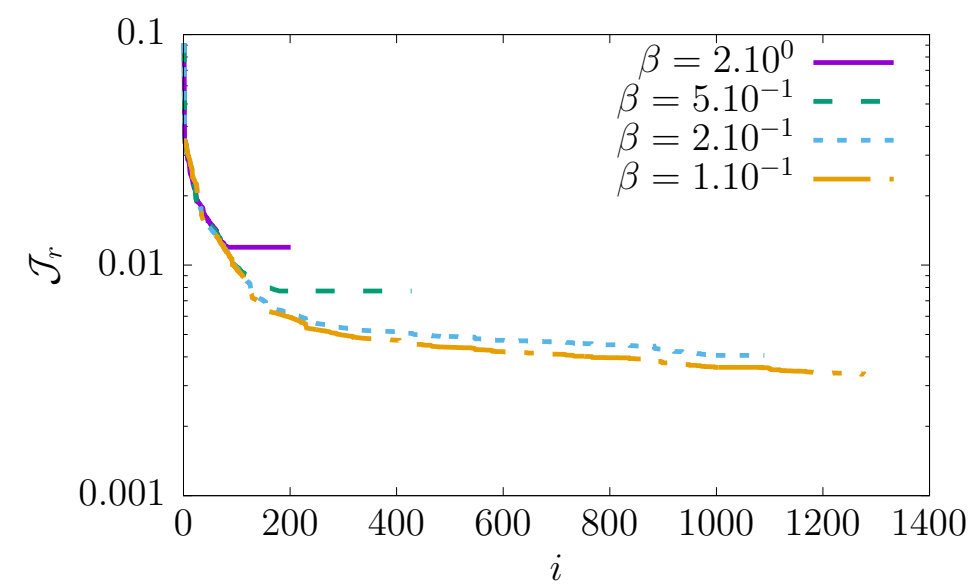

Figure 6: Cost functional $\mathcal{J}_{r}$ as a function of the iteration $i$ during the convergence of the optimisation problem, for various values of wall-distance regularisation parameter $\beta$. 
shape moves away from the initial shape as $\beta$ is decreased.

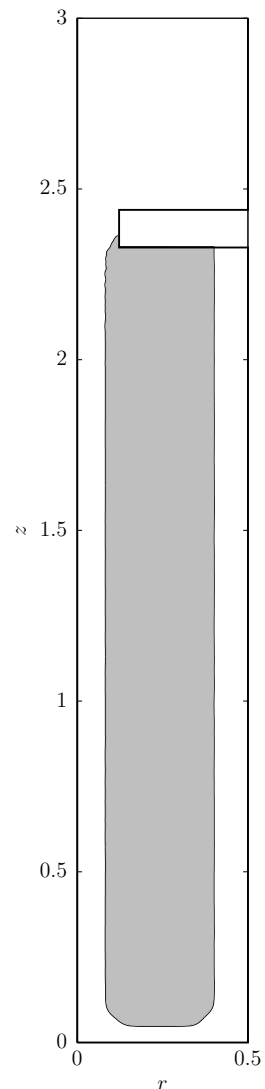

(a) Initial.

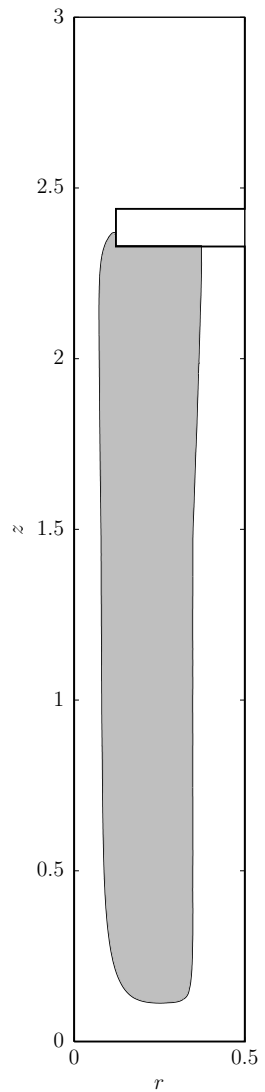

(b) $\beta=2 \cdot 10^{0}$.

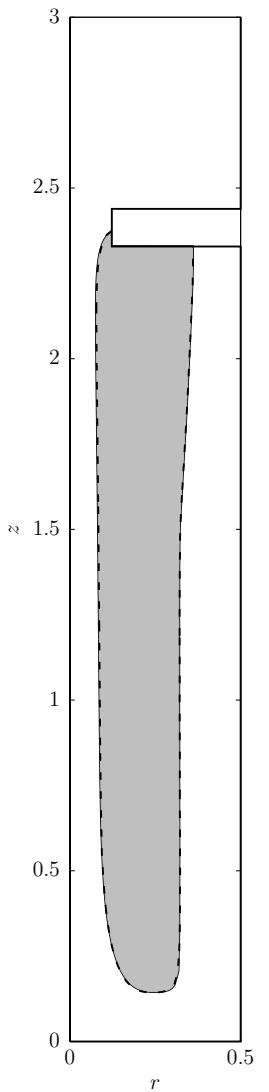

(c) $\beta=5 \cdot 10^{-1}$.

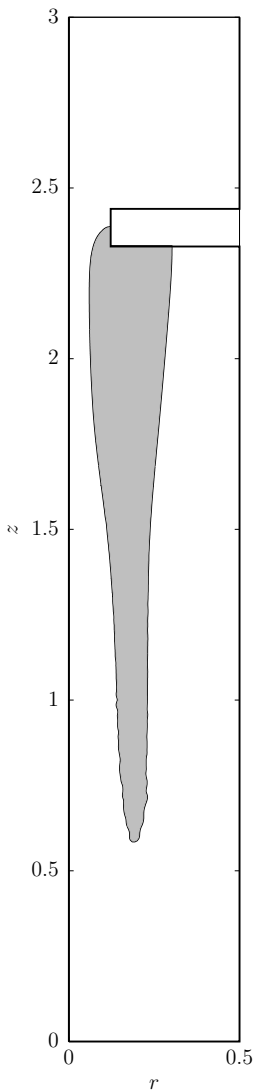

(d) $\beta=2 \cdot 10^{-1}$.

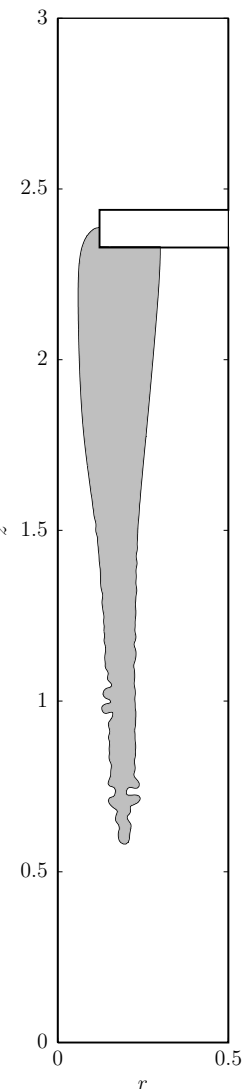

(e) $\beta=1 \cdot 10^{-1}$.

Figure 7: Optimal cavity shapes for different values of the regularisation parameter $\beta$. The gray zones correspond to the immersed body. In $7(\mathrm{c})$, the dashed line is the shape found for a finer mesh.

The numerical convergence is checked for $\beta=5 \cdot 10^{-1}$ using a finer mesh leading to 228569 degrees of freedom, i.e. twice the size of a standard computation, with the same relative mesh stretching. The associated shape, displayed in figure 7 , is accurately preserved.

The resistance, reactance and absorption coefficients are displayed in figure 8. We compare the situation where no immersed wall are considered, 


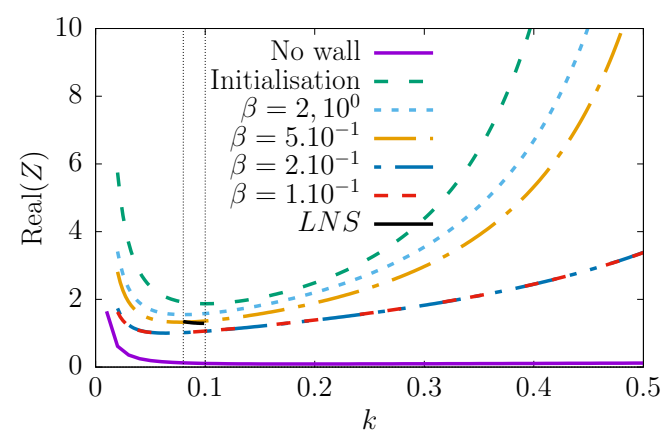

(a) Resistance.

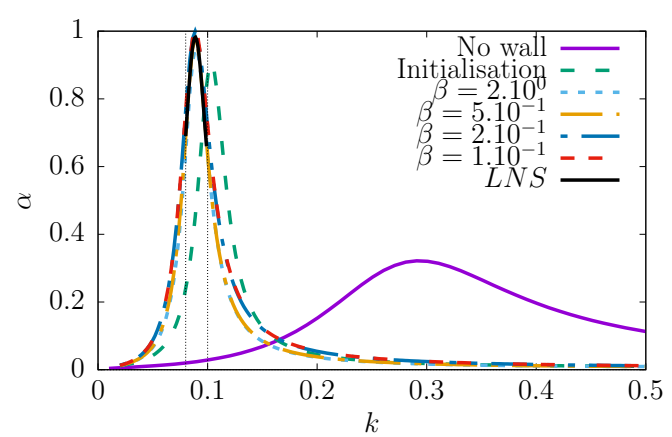

(c) Absorption.

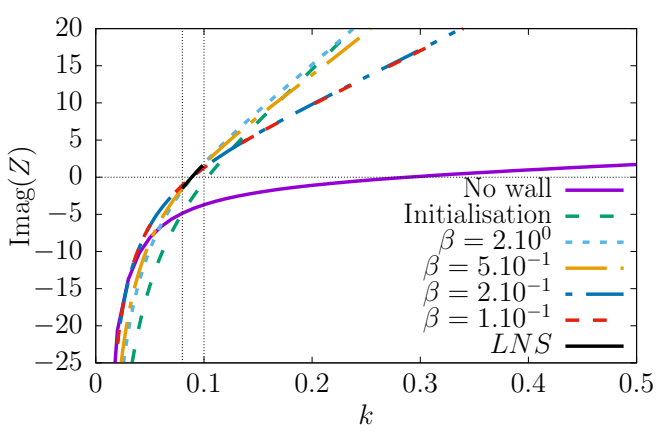

(b) Reactance.

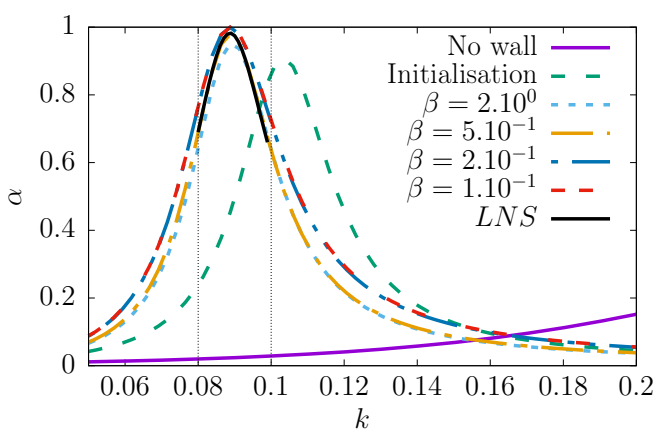

(d) Zoom of the absorption graph.

Figure 8: Performance of the optimal shapes for different values of the regularisation parameter $\beta$. The black line corresponds to the validation with the linearised NavierStokes (LNS) equations using the optimal shape constructed for $\beta=5 \cdot 10^{-1}$.

the initial guess and the optimal shapes. The resonance frequency can be visualised when the reactance vanishes. We can see that all the optimisations quickly bring the resonance frequency in the optimisation frequency range. The shape with no immersed wall has a very low resistance, while the initial guess is too resistive. Relaxing the regularisation $\beta$ allows to bring the resistances closer to one, which leads to high absorptions. We can see that an almost perfect absorption is reached for $\beta \leq 5 \cdot 10^{-1}$ in the desired frequency range.

The attenuation achieved by the optimal shape obtained for $\beta=5 \cdot 10^{-1}$ 
has been validated by performing a simulation using the complete linearised Navier-Stokes equations. For brevity the details of this numerical model are not repeated here but can be found in Billard et al. (2019). The result from this linearised Navier-Stokes model are included in figure 8. This computation requires $1.4 \cdot 10^{6}$ degrees of freedom and has been performed over the target frequency range. The good agreement for both the impedance and the attenuation with the linearised Navier-Stokes model validates the use of the Helmholtz equation with visco-thermal losses for shape optimisation (at least for this application).

Interpreting the cavity as a Helmholtz resonator, we see that the neck and cavity dimensions are adjusted to obtain the desired resonance frequency. The initial shape being too resistive, we see that decreasing $\beta$ leads to an opening of the neck by shaping a smooth horn, while keeping the resonance frequency in the optimisation range. This is indeed done by decreasing the neck length and increasing the cavity volume at the same time. The trend of opening the neck is consistent with the objective of decreasing the resistance. In order to support this hypothesis, figure 9 shows the solution of the linearised Navier-Stokes equations at $k=0.089$ where the absorption is maximum. The velocity magnitude in figure 9 (a) has indeed large values in the neck, while it is negligible in the rest of the cavity. Figure 9(b) also shows the rate of viscous dissipation $\Phi=\tau_{u}: D_{u}$, where $\tau_{u}$ is the viscous shear stress tensor and $D_{u}$ the rate-of-deformation tensor. It shows that the viscous losses take place in the neck in a very thin region near the wall. Firstly, this validates a posteriori the hypothesis made in the Helmholtz model with visco-thermal losses at the boundaries. Secondly, we see that adjusting the 


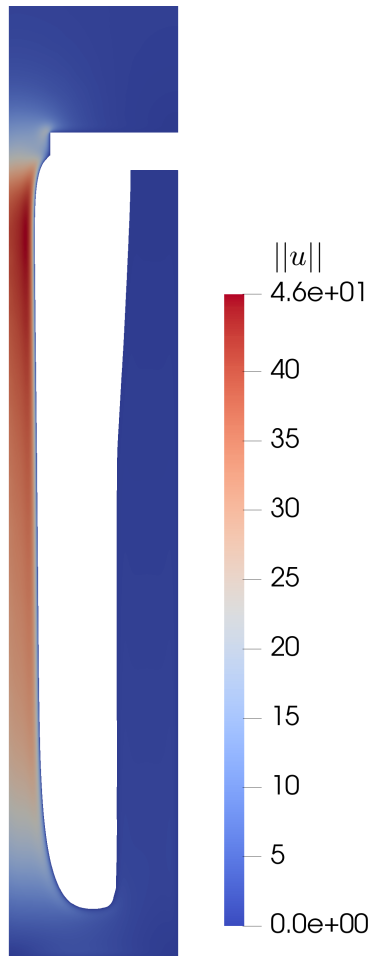

(a) Velocity magnitude $\|\boldsymbol{u}\|$.

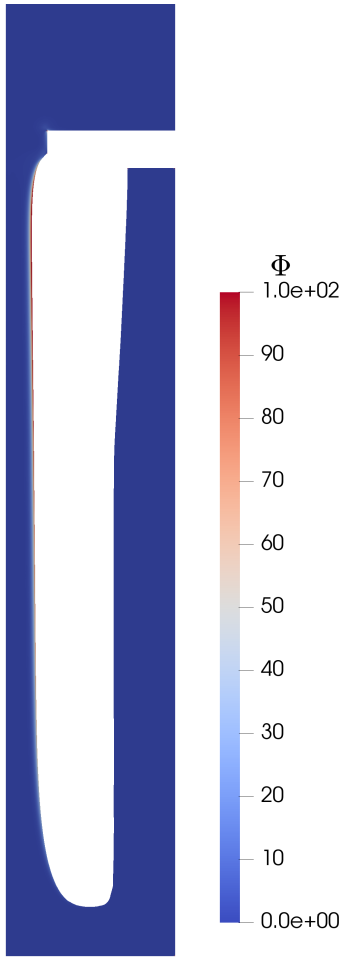

(b) Viscous dissipation $\Phi$.

Figure 9: Solution of the linearised Navier-Stokes equations for $k=0.089$ and $\beta=5 \cdot 10^{-1}$.

neck length allows to obtain the right amount of viscous dissipation to obtain the desired impedance. This is consistent with he physical interpretation made previously to describe the optimisation of the shape.

As can be seen in figure 7 , between $\beta=5 \cdot 10^{-1}$ and $\beta=2 \cdot 10^{-1}$, a quick change of shape brings it far from the initial guess and a further decrease of $\beta$ leads to spurious oscillations of the shape. This illustrates the necessity to regularise this optimisation problem. Looking at the acoustic performance, a regularisation parameter of $\beta=5 \cdot 10^{-1}$ appears in our case to be a good compromise. Of course weight, structural resistance, cost or manufacturing 
considerations can enter into account in the trade-off, or could eventually been incorporated from the beginning in the cost functional. The procedure of penalty parameter selection is classically quantified by L-curve method (Hansen, 1992). This consists in plotting the performance cost functional $\mathcal{J}$ with respect to penalty $\mathcal{J}_{d}=\frac{1}{2} \int_{\Gamma_{\theta}} d_{\Omega_{0}}^{2}(\boldsymbol{x}) \mathrm{d} \boldsymbol{x}$. This curve typically has a " $\mathrm{L}$ " shape, and a fair compromise is to select a value of $\beta$ in the corner of this curve. Unfortunately the curve (not shown here) is a straight line in our case, suggesting to decrease even more the regularisation parameter $\beta$. This is obviously not a reasonable choice with regard to the regularity of the shape figure $7(\mathrm{e})$. A penalty based on the total variation of the shape would certainly produce a L-curve, but it is out of the scope of the present paper.

\section{Conclusion}

In this paper we have demonstrated the possibility of using shape optimisation for the design of absorbing treatments by impedance matching. The use of a model for viscous losses as an equivalent boundary condition allows to use the Helmholtz equation, that is computationally more tractable than employing the full linearised Navier-Stokes equations. The price to pay is the implementation of boundary terms in the shape derivative that involve the wall curvature and second derivatives in space. In that context, the use of the Sobolev gradient as a descent direction improves significantly the regularity of the optimisation problem. In addition, a Tikhonov regularisation allows to penalise the distance of the optimal shape to an initial guess. This improves the well-posedness of the problem and gives to the designer a degree of control to obtain shapes that can be manufactured in the context of 
a local optimisation. For the numerical implementation, a XFEM numerical method has been used where the immersed boundary is tracked by a level set function. A proper level set regularisation gives the possibility at the level-set function to approximate the signed distance function used for the Tikhonov regularisation. This methodology has been employed to optimise the cavity shape of a liner, leading to an almost perfect absorption at a frequency range where standard treatments are inefficient.

\section{Acknowledgements}

The authors gratefully acknowledge the financial support of the Chaire Industrielle MACIA (ANR-16-CHIN-0002) provided by the Safran Group and the Agence Nationale de la Recherche.

\section{Appendix A. Appendix}

Appendix A.1. Derivation of the Lagrangian

The Lagrangian (8) is differentiated with respect to each variable. This appendix details the calculations.

Derivative with respect to $\lambda$.

$$
\left(\nabla_{\lambda} \mathcal{L}, \delta \lambda\right)=0
$$

leads to

$$
\Delta p+k^{2} p=0
$$

Boundary conditions will be enforced later implicitly by using them during the calculation. 
Derivative with respect to $p$ :

$$
\left(\nabla_{p} \mathcal{L}, \delta p\right)=\left(\nabla_{p} \mathcal{J}, \delta p\right)-\int_{k_{1}}^{k_{2}} \operatorname{real}\left[\left(\lambda, \Delta \delta p+k^{2} \delta p\right)\right] \mathrm{d} k
$$

We expand each term separately.

$$
\begin{aligned}
&\left(\nabla_{p} \mathcal{J}, \delta p\right)=\int_{k_{1}}^{k 2} \text { real }\left[j^{*}(p, k) \frac{\partial j(p, k)}{\partial p} \delta p\right] \mathrm{d} k \\
&=\int_{k_{1}}^{k 2} \text { real }\left[\left(\frac{\frac{2}{R^{2}} \int_{\Gamma_{Z}} \delta p \mathrm{~d} l}{e^{-i k L}+e^{i k L}-\bar{p}}\right.\right. \\
&\left.\left.-\frac{\left(e^{-i k L}-e^{i k L}+\bar{p}\right)\left(-\frac{2}{R^{2}} \int_{\Gamma_{Z}} \delta p \mathrm{~d} l\right)}{\left(e^{-i k L}+e^{i k L}-\bar{p}\right)^{2}}\right) j^{*}(p, k)\right] \mathrm{d} k \\
&=\int_{k_{1}}^{k 2} \text { real }\left[\int_{\Gamma_{Z}}\left(\frac{\frac{4}{R^{2}} e^{i k L} j(p, k)}{\left(\left(e^{-i k L}+e^{i k L}-\bar{p}\right)^{2}\right)^{*}}\right)^{*} \delta p \mathrm{~d} l\right] \mathrm{d} k .
\end{aligned}
$$


Besides, we have

$$
\begin{aligned}
& -\int_{k_{1}}^{k_{2}} \operatorname{real}\left[\left(\lambda, \Delta \delta p+k^{2} \delta p\right) \mathrm{d} k\right] \\
= & -\int_{k_{1}}^{k_{2}} \operatorname{real}\left[\left(\Delta \lambda+\left(k^{*}\right)^{2} \lambda, \delta p\right)-\int_{\Gamma_{w}}\left(\nabla \lambda^{*} \cdot \boldsymbol{n}\right) \delta p \mathrm{~d} l+\int_{\Gamma_{w}} \lambda^{*}(\nabla \delta p \cdot \boldsymbol{n}) \mathrm{d} l\right. \\
& -\int_{\Gamma_{Z}}\left(\nabla \lambda^{*} \cdot \boldsymbol{n}\right) \delta p \mathrm{~d} l+\int_{\Gamma_{Z}} \lambda^{*}(\nabla \delta p \cdot \boldsymbol{n}) \mathrm{d} l \\
& \left.-\int_{\Gamma_{s}}\left(\nabla \lambda^{*} \cdot \boldsymbol{n}\right) \delta p \mathrm{~d} l+\int_{\Gamma_{s}} \lambda^{*}(\nabla \delta p \cdot \boldsymbol{n}) \mathrm{d} l\right] \mathrm{d} k \\
= & -\int_{k_{1}}^{k_{2}} \operatorname{real}\left[\left(\Delta \lambda+\left(k^{*}\right)^{2} \lambda, \delta p\right)+\int_{\Gamma_{Z}}-\left(\nabla \lambda^{*} \cdot \boldsymbol{n}\right) \delta p+\left(i k^{*} \lambda\right)^{*} \delta p \mathrm{~d} l\right. \\
& \left.+\int_{\Gamma_{s}}-\left(\nabla \lambda^{*} \cdot \boldsymbol{n}\right) \delta p \mathrm{~d} l\right] \mathrm{d} k \\
& +\int_{\Gamma_{w}}\left(-(\nabla \lambda \cdot \boldsymbol{n})+\delta_{v} \frac{-i-1}{2} \Delta_{T} \lambda-\delta_{T}\left(k^{*}\right)^{2} \frac{(-i-1)(\gamma-1)}{2} \lambda\right)^{*} \delta p \mathrm{~d} l .
\end{aligned}
$$

We deduce the adjoint equation

$$
\begin{cases}\Delta \lambda+\left(k^{*}\right)^{2} \lambda=0 & \boldsymbol{x} \in \Omega \\ \nabla \lambda . \boldsymbol{n}+\delta_{v} \frac{i+1}{2} \Delta_{T} \lambda-\delta_{T}\left(k^{*}\right)^{2} \frac{(i+1)(\gamma-1)}{2} \lambda=0 & \boldsymbol{x} \in \Gamma_{w} \\ \nabla \lambda . \boldsymbol{n}-i k^{*} \lambda=\frac{4}{R^{2}} \frac{e^{i k L} j(p, k)}{\left(\left(e^{-i k L}+e^{i k L}-\bar{p}\right)^{2}\right)^{*}} & \boldsymbol{x} \in \Gamma_{Z} \\ \nabla \lambda . \boldsymbol{n}=0 & \boldsymbol{x} \in \Gamma_{s} .\end{cases}
$$

Appendix A.2. Weak formulation of the level set regularisation

We detail the weak formulation associated with the level-set regularisation equation (37). A SUPG numerical scheme is employed, leading to the 
following expression

$$
\begin{aligned}
& \int_{\Omega} \frac{\partial \psi}{\partial t}\left(w-\tau \operatorname{sign}\left(\psi_{0}\right) \boldsymbol{n} \cdot \nabla w\right) \mathrm{d} \boldsymbol{x}+\int_{\Omega} \operatorname{sign}\left(\psi_{0}\right)|\nabla \psi| w \mathrm{~d} \boldsymbol{x} \\
& +\tau \int_{\Omega}(\nabla \psi \cdot \boldsymbol{n})(\nabla w \cdot \boldsymbol{n}) \mathrm{d} \boldsymbol{x}+\nu \int_{\Omega} \nabla w \cdot\left(d_{p}(|\nabla \psi|) \nabla \psi\right) \mathrm{d} \boldsymbol{x} \\
& -\nu \int_{\Gamma} d_{p}(|\nabla \psi|) \nabla \psi \cdot \boldsymbol{n} w \mathrm{~d} l+\nu \tau \operatorname{sign}\left(\psi_{0}\right) \int_{\Omega} \operatorname{div}\left(d_{p}(|\nabla \psi|) \nabla \psi\right) \nabla w \cdot \boldsymbol{n} \mathrm{d} \boldsymbol{x} \\
& =\int_{\Omega} \operatorname{sign}\left(\psi_{0}\right) w \mathrm{~d} \boldsymbol{x}-\tau \int_{\Omega} \nabla w \cdot \boldsymbol{n} \mathrm{d} \boldsymbol{x} .
\end{aligned}
$$

Let us remark that every terms containing $\operatorname{sign}\left(\psi_{0}\right)$ have to be assembled in two separated parts: for $\boldsymbol{x} \in \Omega$ and for $\boldsymbol{x} \in\left(\mathcal{D} \backslash\left(\Omega \cup \Gamma_{\theta}\right)\right)$.

For the time advancement, an implicit Euler scheme is used. At the time step $i$, the convection direction $\boldsymbol{n}=-\frac{\nabla \psi}{|\nabla \psi|}$ and the diffusion coefficient $p(|\nabla \psi|)$ are expressed at the time step $i$, while the other terms are expressed at the time $i+1$ :

$$
\begin{aligned}
& \int_{\Omega} \psi^{(i+1)}\left(w-\tau \operatorname{sign}\left(\psi_{0}\right) \boldsymbol{n}^{(i)} \cdot \nabla w\right) \mathrm{d} \boldsymbol{x}-\Delta t \int_{\Omega} \operatorname{sign}\left(\psi_{0}\right)\left(\nabla \psi^{(i+1)} \cdot \boldsymbol{n}^{(i)}\right) w \mathrm{~d} \boldsymbol{x} \\
& +\Delta t \tau \int_{\Omega}\left(\nabla \psi^{(i+1)} \cdot \boldsymbol{n}^{(i)}\right)\left(\nabla w \cdot \boldsymbol{n}^{(i)}\right) \mathrm{d} \boldsymbol{x}+\Delta t \nu \int_{\Omega} \nabla w \cdot\left(d_{p}\left(\left|\nabla \psi^{(i)}\right|\right) \nabla \psi^{(i+1)}\right) \mathrm{d} \boldsymbol{x} \\
& -\Delta t \nu \int_{\Gamma} d_{p}\left(\left|\nabla \psi^{(i)}\right|\right) \nabla \psi^{(i+1)} \cdot \boldsymbol{n}^{(i)} w \mathrm{~d} l \\
& +\Delta t \nu \tau \operatorname{sign}\left(\psi_{0}\right) \int_{\Omega} \operatorname{div}\left(d_{p}\left(\left|\nabla \psi^{(i)}\right|\right) \nabla \psi^{(i+1)}\right) \nabla w \cdot \boldsymbol{n}^{(i)} \mathrm{d} \boldsymbol{x} \\
& =\int_{\Omega} \psi^{(i)}\left(w-\tau \operatorname{sign}\left(\psi_{0}\right) \boldsymbol{n}^{(i)} \cdot \nabla w\right) \mathrm{d} \boldsymbol{x}+\Delta t \int_{\Omega} \operatorname{sign}\left(\psi_{0}\right) w \mathrm{~d} \boldsymbol{x} \\
& -\Delta t \tau \int_{\Omega} \nabla w \cdot \boldsymbol{n}^{(i)} \mathrm{d} \boldsymbol{x} .
\end{aligned}
$$




\section{References}

Achtziger, W., Kočvara, M., 2007. Structural topology optimization with eigenvalues. SIAM Journal on Optimization 18 (4), 1129-1164.

Allaire, G., De Gournay, F., Jouve, F., Toader, A.-M., 2005. Structural optimization using topological and shape sensitivity via a level set method. Control and Cybernetics 34 (1), 59.

Allaire, G., Jouve, F., Michailidis, G., 2016. Thickness control in structural optimization via a level set method. Structural and Multidisciplinary Optimization 53 (6), 1349-1382.

Allaire, G., Jouve, F., Toader, A.-M., 2004. Structural optimization using sensitivity analysis and a level-set method. Journal of Computational Physics 194 (1), 363-393.

Andersen, P. R., Henríquez, V. C., Aage, N., 2019. Shape optimization of micro-acoustic devices including viscous and thermal losses. Journal of Sound and Vibration.

Armijo, L., 1966. Minimization of functions having Lipschitz continuous first partial derivatives. Pacific Journal of Mathematics 16 (1), 1-3.

Bängtsson, E., Noreland, D., Berggren, M., 2003. Shape optimization of an acoustic horn. Computer Methods in Applied Mechanics and Engineering 192 (11-12), 1533-1571.

Berggren, M., Bernland, A., Noreland, D., 2018. Acoustic boundary layers as boundary conditions. Journal of Computational Physics 371, 633 - 650 . 
Bernland, A., Wadbro, E., Berggren, M., 2018. Acoustic shape optimization using cut finite elements. International Journal for Numerical Methods in Engineering 113 (3), 432-449.

Billard, R., Tissot, G., Gabard, G., Versaevel, M., Groby, J.-P., 2019. A mode matching model for the impedence of perforated plate liners. In: 25th AIAA/CEAS Aeroacoustic Conference and Exhibit. 20-23 May, Delft, Neetherlands, AIAA 2019-2702.

Bossart, R., Joly, N., Bruneau, M., 2003. Hybrid numerical and analytical solutions for acoustic boundary problems in thermo-viscous fluids. Journal of Sound and Vibration 263 (1), $69-84$.

Burman, E., 2010. Ghost penalty. Comptes Rendus Mathematique 348 (2122), $1217-1220$.

Burman, E., Claus, S., Hansbo, P., Larson, M. G., Massing, A., 2015. CutFEM: discretizing geometry and partial differential equations. International Journal for Numerical Methods in Engineering 104 (7), 472-501.

Caeiro, F., Sovardi, C., Förner, K., Polifke, W., 2017. Shape optimization of a Helmholtz resonator using an adjoint method. International Journal of Spray and Combustion Dynamics 9 (4), 394-408.

Desai, J., Faure, A., Michailidis, G., Parry, G., Estevez, R., 2018. Topology optimization in acoustics and elasto-acoustics via a level-set method. Journal of Sound and Vibration 420, 73-103.

Díaaz, A. R., Kikuchi, N., 1992. Solutions to shape and topology eigenvalue 
optimization problems using a homogenization method. International Journal for Numerical Methods in Engineering 35 (7), 1487-1502.

Donea, J., Huerta, A., 2003. Finite element methods for flow problems. John Wiley \& Sons.

Dühring, M. B., Jensen, J. S., Sigmund, O., 2008. Acoustic design by topology optimization. Journal of Sound and Vibration 317 (3-5), 557-575.

Hansen, P. C., 1992. Analysis of discrete ill-posed problems by means of the L-curve. SIAM review 34 (4), 561-580.

Jameson, A., 2003. Aerodynamic shape optimization using the adjoint method. Lectures at the Von Karman Institute, Brussels.

Jensen, J. S., Sigmund, O., 2005. Topology optimization of photonic crystal structures: a high-bandwidth low-loss T-junction waveguide. Journal of Optical Soscity of America B 22 (6), 1191-1198.

Legrain, G., Chevaugeon, N., Dréau, K., 2012. High order X-FEM and levelsets for complex microstructures: uncoupling geometry and approximation. Computer Methods in Applied Mechanics and Engineering 241, 172189.

Li, C., Xu, C., Gui, C., Fox, M. D., 2010. Distance regularized level set evolution and its application to image segmentation. IEEE Transactions on Image Processing 19 (12), 3243-3254.

Ma, Z.-D., Cheng, H.-C., Kikuchi, N., 1994. Structural design for obtain- 
ing desired eigenfrequencies by using the topology and shape optimization method. Computing Systems in Engineering 5 (1), 77-89.

Moës, N., Dolbow, J., Belytschko, T., 1999. A finite element method for crack growth without remeshing. International Journal for Numerical Methods in Engineering 46 (1), 131-150.

Osher, S., Fedkiw, R. P., 2001. Level set methods: an overview and some recent results. Journal of Computational physics 169 (2), 463-502.

Protas, B., Bewley, T. R., Hagen, G., 2004. A computational framework for the regularization of adjoint analysis in multiscale PDE systems. Journal of Computational Physics 195 (1), 49-89.

Sonntag, M., Schmidt, S., Gauger, N. R., 2016. Shape derivatives for the compressible Navier-Stokes equations in variational form. Journal of Computational and Applied Mathematics 296, 334-351.

Tikhonov, A. N., Arsenin, V. Y., 1977. Solution of ill-posed problems. Winston \& Sons.

Udawalpola, R., Berggren, M., 2008. Optimization of an acoustic horn with respect to efficiency and directivity. International Journal for Numerical Methods in Engineering 73 (11), 1571-1606.

Udawalpola, R., Wadbro, E., Berggren, M., 2011. Optimization of a variable mouth acoustic horn. International Journal for Numerical Methods in Engineering 85 (5), 591-606. 
Wadbro, E., Udawalpola, R., Berggren, M., 2010. Shape and topology optimization of an acoustic horn-lens combination. Journal of Computational and Applied Mathematics 234 (6), 1781-1787.

Wang, F., Jensen, J. S., Sigmund, O., 2011. Robust topology optimization of photonic crystal waveguides with tailored dispersion properties. Optical Society of America. Journal B: Optical Physics 28 (3), 387-397. 